\title{
Evaluation of excitation energy and spin in fission fragments using the statistical model, and the FIPPS project
}

\author{
H. Faust ${ }^{1}$, G. Kessedjian ${ }^{2}$, C. Sage ${ }^{2}$, U. Koester ${ }^{1}$, and A. Chebboubi ${ }^{1,2}$ \\ ${ }^{1}$ Institut Laue-Langevin, 38042 Grenoble, France \\ ${ }^{2}$ LPSC, CNRS/IN2P3, UJF Grenoble1, INPG, 38042 Grenoble, France
}

\begin{abstract}
We review the statistical model and its application for the process of nuclear fission. The expressions for excitation energy and spin distributions for the individual fission fragments are given. We will finally emphasize the importance of measuring prompt gamma decay to further test the statistical model in nuclear fission with the FIPPS project.
\end{abstract}

\section{Introduction}

Despite considerable efforts since the discovery of nuclear fission about 73 years ago a coherent theory of the process is still missing. Two antagonistic approaches have been formulated to estimate mass and nuclear charge distributions for a fissioning system, and the distribution functions for fragment excitation and spin. The general accepted approach to calculate mass and charge distribution functions view the process of fission as a sequence of liquid drop shapes. Calculations proceed by including numerous degrees of freedom do describe fission configurations from saddle to scission. Shell corrections calculated for each deformation step lead to structures in the potential energy surfaces, and the most probable fission configuration is determined by following the steepest descend in this multidimensional potential energy landscape. In general only the energy of the ground-state is needed in this approach, and there is no dependence on level densities.

In contrast to this geometric approach is the statistical model in nuclear physics. Here the decay process is viewed as a one step process, from the initial to the final state. The probability for decay is exclusively determined by the density of levels which are attainable in the decay. In the statistical decay process all nuclear levels carry the same weight, independent of the quantum numbers and parity which characterize the excited levels. The use of the statistical approach is generally justified for regions for high level density, where the individuality of quantum states is washed out by the use of a large number of them.

It is well known that mass and charge distribution functions for fragments from fissioning systems cannot be described by the statistical model, unless selection rules are at work which picks out individual levels for the fission process. In contrast to mass and charge distribution functions where the statistical model fails to reproduce the experimental data, there is considerable agreement when excitation energy and spin distributions are calculated by using the statistical model. We will in the following develop the statistical model description for excitation energy and spin in fragments. Finally we will present a new spectrometer which will allow to test important aspects of fission decay with respect to the statistical model. 


\section{Excitation energy and spin distributions in fission fragments}

In order to obtain a coherent picture of the fission reaction we assume that the process can be separated into distribution functions for mass and nuclear charge on one hand, and for excitation energy and spin on the other hand. In the following the mass and charge distribution problem is discarded. The probability for fission from a compound system $\left(A_{c}, Z_{c}\right)$ into the fragment pairs $\left(A_{1}, Z_{1}, A_{2}, Z_{2}\right)$ with masses $A_{i}$ and nuclear charges $Z_{i}$ for the fragments is given by

$$
W \propto \emptyset\left(A_{1}, Z_{1}, A_{2}, Z_{2}\right) \cdot \theta\left(E_{1}^{*}, J_{1}, E_{2}^{*}, J_{2}\right)
$$

The distribution functions for excitation energy $E^{*}$ and spin $J$ for both fragments is given by $\theta\left(E_{1}^{*}, J_{1}, E_{2}^{*}, J_{2}\right)$. The aim of the present paper is to formulate this distribution functions on the basis of the thermo-dynamical model and to determine the observable in nuclear fission which allows for a verification of this assumption.

\subsection{Excitation energy and spin distributions in fission fragments}

\subsubsection{The role of entropy and thermodynamics}

Starting from the second law of thermodynamics a decay proceeds because the entropy in the process increases. The probability for the decay is

$$
W \sim e^{S}
$$

where $\mathrm{S}$ is the entropy. In nuclear physics the entropy is connected to the density of available states in an energy interval which can be reached in the decay. The entropy for a nuclear decay is given by

$$
S=\ln \rho
$$

with $\rho$ being the level density at excitation energy $E^{*}$. Inserting this expression in Eq. (2) leads to the well known expression where the probability of decay is directly proportional to the level density of excited states in the final nucleus

$$
W \sim \rho\left(E^{*}\right)
$$

The most basic expression for the nuclear level density is given by Fermi

$$
\rho\left(E^{*}\right)=\exp \left(2 \sqrt{a \cdot E^{*}}\right)
$$

with $a$ being the level density parameter. This function is a strongly increasing function of the excitation energy of the nucleus. The level density parameter depends on the mass and nuclear charge on the fragment, and is mostly calculated as a smooth function of the nuclear mass on top of which a shell correction term is added, ref. [1]. Level density parameter as function of mass show the characteristic saw tooth behaviour which is determined by magic shells where the shell corrections are strong and the level density parameter exhibit a minimum value. 
To proceed further with the expression in Eq. (4) we apply basic thermodynamics. The statistical ensemble to be used is the micro-canonical ensemble, which is defined for non interacting nuclei. In a concrete example these may be all ${ }^{142} \mathrm{Ba}$ fission products from thermal neutron induced fission of ${ }^{235} U$. These Barium nuclei are non-interacting, and are not correlated in time and space, but they form a statistical ensemble where the excitation can be described by a single value of the temperature $k T$. The thermodynamic model cuts in the strong increasing function given by Eq. (4) with the Boltzmann-term $\exp \left(-\frac{E^{*}}{k T}\right)$, with $k T$ being the temperature of the nuclear ensemble. The final expression for the decay probability reads

$$
W \sim \exp \left(-\frac{E^{*}}{k T}\right) \cdot \rho\left(E^{*}\right)
$$

The distribution of the excitation energy in the fragments of the statistical ensemble is given by Eq. (6), which represents a bell shape curve where the maximum is at $\left\langle E^{*}\right\rangle=a \cdot k T^{2}$. The constant $k$ is taken to be 1 in order to have the dimensions of the temperature $T$ in $[\mathrm{MeV}]$.

In order to evaluate Eq. (6) the temperature of the statistical ensemble of fission products must be known. There are in principle 3 ways to proceed. The first one assumes that one is able to calculate the value of the temperature from the total excitation energy distribution TXE of the statistical ensemble. It demands, however, that a decay law can be formulated which would lead to this distribution. In nuclear fission this is not known. The second possibility is the formulation of an empirical law which connects the temperature value to other observable in the fission process, like the $Q$-value, or the mass and charge of the fissile compound system. We will show later that such a formulation exists. A third evident possibility for the determination of the temperature of the statistical ensemble is by a measurement of the total kinetic energy, or the single kinetic energy of a fission product and the use of energy and momentum conservation laws. We observe here that in using the statistical model only excitation energies of the fragments are provided. However, applying energy and momentum conservation to a known TXE distribution leads to the distribution functions for single fragment kinetic energies, ref. [2].

\subsubsection{The expressions for excitation energy and spin distributions of fission products}

A complete level density expression goes farther than the simple expression provided by Fermi, it includes too the value of the spin on the nuclear levels. The first attempt for the counting of nuclear levels in the dependence of the spin value in the nuclear potential was done by Bethe, who demonstrated that the total level density can be separated in a term for the excitation energy, and a second term describing the spin distribution. The total level density expression reads

$$
\rho\left(E^{*}, J\right)=f\left(E^{*}\right) \cdot g(J)
$$

The function $f\left(E^{*}\right)$ can be approximated by Fermi, Eq. (5). Bethe has shown that in a shell model potential the spin distribution in the level density is given by

$$
g(J)=(2 J+1) \exp \left(\frac{-J(J+1)}{2 \sigma^{2}}\right)
$$

The dependencies on fission physics are included in the spin cut off parameter $\sigma$

$$
\sigma^{2}=0.0888 \cdot a \cdot k T \cdot A^{2 / 3}
$$

We note that the spin distributions, as well as the excitation energy distributions, exhibit a structure as function of fragment mass which is imposed by the level density parameter.

Finally we arrive at four equations which perfectly describe excitation energy and spin distribution for fragment 1 and fragment 2. From these distribution functions the entry states of fission fragments are constructed, and their neutron and gamma decay can be calculated by using the statistical model known from compound nuclear reactions. 


$$
\begin{aligned}
f_{1}\left(E^{*}\right) d E^{*} & \propto \exp \left(-\frac{E^{*}}{k T}\right) \cdot \rho_{1}\left(E^{*}\right) d E^{*} \\
f_{2}\left(E^{*}\right) d E^{*} & \propto \exp \left(-\frac{E^{*}}{k T}\right) \cdot \rho_{2}\left(E^{*}\right) d E^{*} \\
g_{1}(J) & \propto \frac{2 J+1}{2 \sigma_{1}^{2}} \exp \left(-\frac{J(J+1)}{2 \sigma_{1}^{2}}\right) \\
g_{2}(J) & \propto \frac{2 J+1}{2 \sigma_{2}^{2}} \exp \left(-\frac{J(J+1)}{2 \sigma_{2}^{2}}\right)
\end{aligned}
$$

These equations hold for fission fragment 1 and 2 , respectively. The temperature $k T$ of both fragments is the same, however the distributions for excitation energy and spin may be very different because the level density parameter for the two fragments differ from each other. Also the value of the mean fragment spin of both fragments may be different, indicating that, on the basis of angular momentum conservation, any residual angular momentum has to be found in orbital momentum between the fragments at the moment of emission.

\subsection{The temperature of the micro-canonical ensemble}

The temperature parameter for the micro-canonical ensemble of one kind of fission products is the only thermodynamic variable present in the theory. The value for the temperature is calculated from a given total excitation energy of the combined system, by taking the derivative of the level density at this excitation

By using the Fermi-gas expression this gives

$$
\frac{1}{k T}=\frac{d(\ln \rho)}{d T X E}
$$

$$
k T=\sqrt{\frac{T X E}{a_{1}+a_{2}}}
$$

Provided the distribution function of the total excitation energy TXE for a given fission product is known, it is possible to deduce the temperature by taking the derivative at the maximum of the distribution. If, by measurement, a single fragment kinetic energy value is known, the application of energy and momentum conservation for a binary reaction leads to the temperature value to be

$$
k T=\sqrt{\frac{Q-E_{k i n}^{1}\left[1+\frac{A_{1}}{A_{2}}\right]}{a_{1}+a_{2}}}
$$

This includes the measurement of the kinetic energy of fragment 1 and the $Q$-value of the reaction. In this case the calculation of energy and spin distributions is totally parameter-free.

In the event that nothing is known on the total excitation energy distribution or the single fragment energy, we have found an empirical relationship which relates the temperature of the statistical ensemble for one kind of fragments to the $Q$-value of the reaction, $k T=\bar{f} \cdot Q$. The value of the nuclear temperature is directly proportional to the $Q$-value of the reaction. We have shown that, within a limited region for the actinides, the constant $\bar{f}$ is a function of the nuclear charge of the compound system only, with $\bar{f}=c Z_{c}+b$. The constants have values of $c=3.29 \cdot 10^{-4}$ and $b=-0.0258$ for actinides in between Actinium and Californium, ref. [3]. A theoretical justification for this simple behaviour for $k T$ is, however, missing.

\section{A new spectrometer to verify thermodynamic model predictions for nuclear fission}

We propose a new spectrometer to get access to the values of fission observable predicted by the thermodynamic model. To test the validity of the thermodynamic description neutron and gamma decay data are needed to reconstruct excitation energy and spin of the entry states given by Eqs. $10 a, b, c, d$. Prompt gamma decay and the population of isomeric states and ground-state band members has to be measured for each fission fragment characterized in $(A, Z, k T)$. With the new spectrometer mass $A$ and nuclear charge $Z$ of the fission product are to be determined via the 
measurement of characteristic $\gamma$-ground-state transitions or other strong $\gamma$-transition near the Yrastline. The temperature is determined by measurement of the single fragment kinetic energy, and the application of conservation laws.

The identification of $\gamma$-rays of fragments belonging to one statistical ensemble is, however, not an easy task. These $\gamma$-rays are in general hidden in the huge background of $\gamma$-decays from all the other fission products. Therefore a filter has to be applied to select the right mass and nuclear charge of the fission product in question. We think that such a filter with high efficiency and focussing characteristics is best provided by the use of a gas filled spectrometer, coupled to a high efficient $\gamma$ ray array. The lay-out of such a spectrometer is shown in fig. 1. Basically a fissile thin target layer is positioned in a neutron beam. One of the fission products will leave the target and the velocity will be measured by a time of flight device. Then the fission fragment will enter a gas filled magnet section and will be analysed in magnetic rigidity and energy loss, giving mass, nuclear charge, and kinetic energy. The second fission product will be captured in the thick backing behind the target layer and its gamma decay is measured Doppler-shift free with the $\gamma$-array. The velocity measurement together with the mass determination will enable to calculate the temperature of the statistical ensemble. Actually the characteristics of a gas filled magnet are investigated at the ILL.

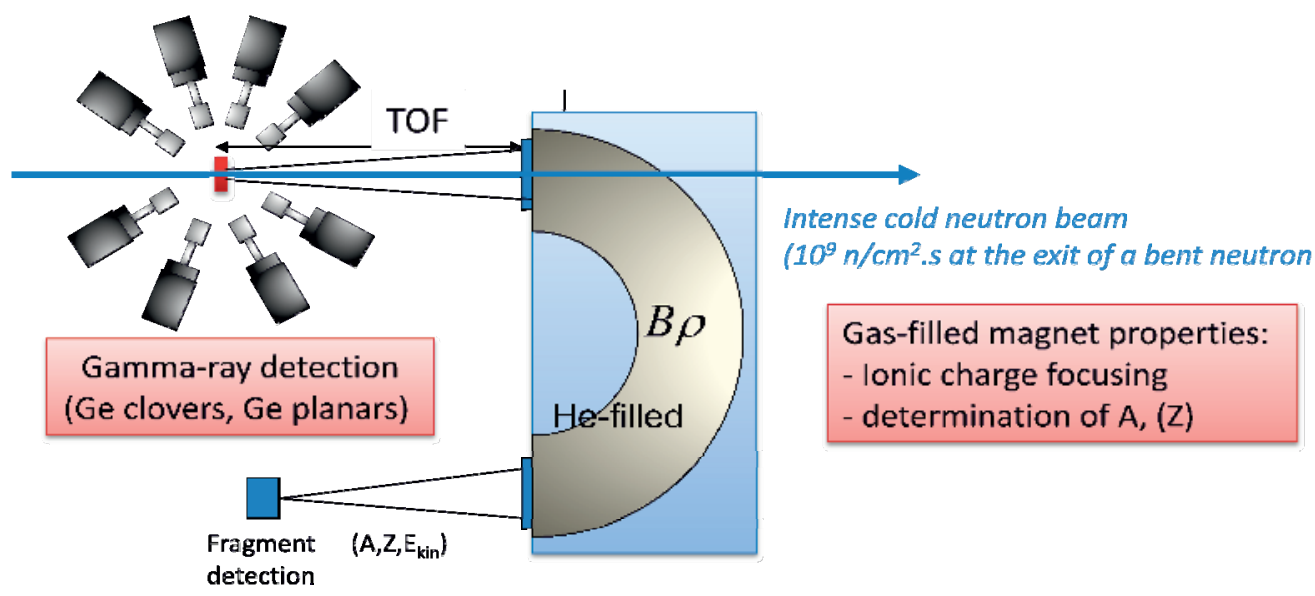

Fig. 1. The FIPPS-spectrometer for the test of the thermo-dynamical model in fission

\section{References}

1. A. Gilbert and A.G.W. Cameron, Can. J. of Phys. 43, 1446 (1965)

2. H. Faust, Seminar on fission, C.Wagemans, J. Wagemans and P. D'hont eds., World Scientific, 2011, p. 89.

3. H. Faust, U. Koester, T. Materna and W. Urban, J. of the Korean Phys. Society, 59879 (2011) 